\title{
Joint constraints on the galaxy cluster pressure profile from Planck and SPT-SZ
}

\author{
Jean-Baptiste Melin ${ }^{1, *}$, Gabriel W. Pratt ${ }^{1,2}$, and Monique Arnaud ${ }^{1,2}$ \\ ${ }^{1}$ IRFU, CEA, Université Paris-Saclay, F-91191 Gif-sur-Yvette, France \\ ${ }^{2}$ Université Paris Diderot, AIM, Sorbonne Paris Cité, CEA, CNRS, F-91191, Gif-sur-Yvette, France
}

\begin{abstract}
We present the average pressure profile of 461 galaxy clusters from the 2500d South Pole Telescope catalogue measured in the Planck and SPT-SZ data jointly. The joint measurement is performed in Fourier space for the first time, and takes advantage of the complementarity between the high resolution of SPT (1.75 arcmin beam) and the coverage of large angular scales by Planck (>30 arcmin). The redshift leverage of the catalogue allows the study of the evolution of the profile. We also constrain the profile shape in the outskirts.
\end{abstract}

\section{Introduction}

The thermal Sunyaev-Zeldovich (SZ) effect $[1,2]$ is the inverse Compton scattering of the Cosmic Microwave Background photons by hot electrons in galaxy clusters. It depends on the Compton $y$-parameter

$$
y=\frac{\sigma_{\mathrm{T}}}{m_{\mathrm{e}} c^{2}} \int_{l o s} P_{e} d l
$$

where $\sigma_{\mathrm{T}}$ is the Thomson cross-section, $m_{\mathrm{e}}$ is the electron rest mass, $c$ is the speed of light light and $P_{e}=n_{e} \times T_{e}$ is the pressure (the product of the electronic density and temperature). The integral is performed along the line-of-sight $(l o s)$. Observing the SZ effect in galaxy clusters allows one to probe directly their electronic pressure profiles. Structure formation in the Universe being dominated by the gravitational interaction on the largest scales, the pressure profiles are expected to be self-similar: they can be rescaled to a unique profile ('the universal pressure profile') according to the cluster mass and redshift. The pressure profile can provide a wealth of information on structure formation, in particular via its evolution with redshift, or in studying the assembly process in the cluster outskirts.

The South Pole Telescope (SPT) collaboration released recently the data of the SPT-SZ survey [3]. This dataset covers approximately $2,500 \mathrm{deg}^{2}$ and is complementary to the Planck all-sky dataset in terms of resolution and frequency coverage. The goal of this work is to combine the two data sets and average the profiles of the clusters detected in the SPT-SZ survey to improve the signal-to-noise and radial resolution of the measured average pressure profile. We also study the evolution of the average pressure profile and examine the outskirt region. The datasets are presented in Sect. 2, the analysis method in Sect. 3 and the results in Sect. 4. We conclude in Sect. 5.

\footnotetext{
*e-mail: jean-baptiste.melin@cea.fr
} 


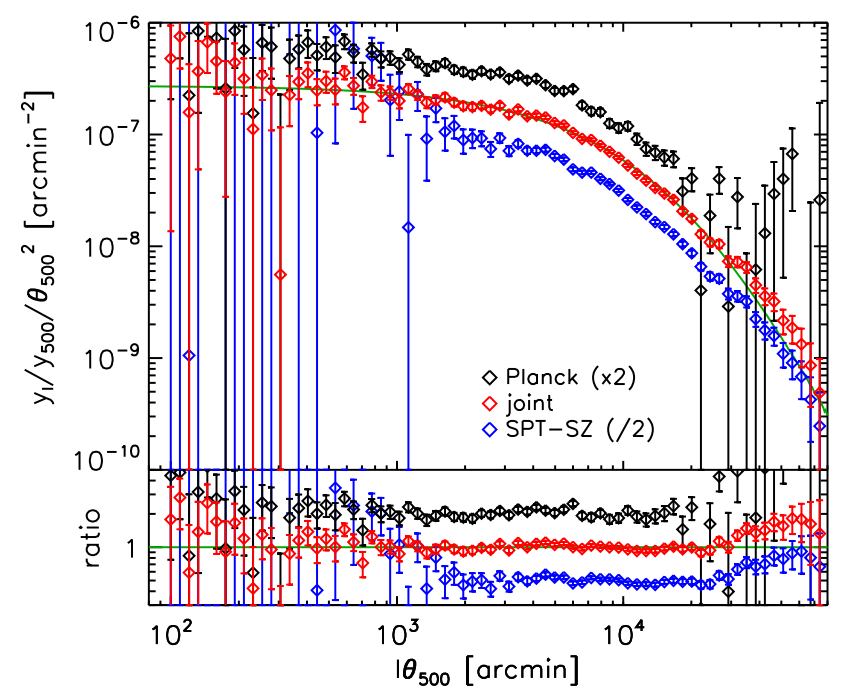

Figure 1. Average pressure profile of the 461 SPT-SZ clusters in harmonic space. The black, red and blue diamonds correspond to the Planck only, Planck+SPT-SZ and SPT-SZ only analysis respectively. The black (resp. blue) diamonds have been multiplied (resp. divided) by two for clarity. The solid green line is the PIPV profile, which is in very good agreement with the average pressure profile.

\section{Datasets}

We work with the fiducial SPT-SZ 2500d cluster catalogue published by the SPT collaboration [4], which contains 677 candidates. We keep only the candidates with assigned redshift, and we remove the objects located at the boundary or outside the SPT-SZ public footprint, or having a SPT-SZ flux $Y^{0.75} \geqslant 2 \times 10^{-4} \mathrm{arcmin}^{2}$, for which the filter transfer function is not accurate [Fig. B.3. of 5]. After this selection, our sample contains 461 clusters. The cluster mass $M_{500}{ }^{1}$ is provided with the catalogue; these values were renormalised by a factor of 0.8 to make them compatible with the mass definition adopted by Arnaud et al. [6], and the corresponding radius $R_{500}$ and angular size $\theta_{500}$ were then obtained.

We use the six Planck public all sky maps of the High Frequency Instrument $(100,143$, $217,353,545,857 \mathrm{GHz}$ ). We upgraded the pixel size from HEALPix [7] $N_{\text {side }}=2048$ to $N_{\text {side }}=8192(0.43 \mathrm{arcmin})$ and rotated the coordinate system from galactic to equatorial to make the format compatible with the SPT-SZ data [details given in 5]. We also used the three SPT-SZ only public maps at 90, 150, $200 \mathrm{GHz}$ [3].

\section{Method}

The observed clusters were convolved by the instrumental point spread functions (PSF) of the experiments and also by the filter transfer function (FTF) for SPT. Averaging clusters requires radial rescaling of the profiles by $\theta_{500}$. To perform the cleanest possible PSF and FTF deconvolution across cluster sizes, we performed our analysis in Fourier space. We also use the flat sky approximation to directly map the Fourier (wavenumber $k$ ) to the harmonic

\footnotetext{
${ }^{1}$ mass enclosed in a sphere of radius $R_{500}$ whose mean density is 500 times the critical density at the cluster redshift.
} 


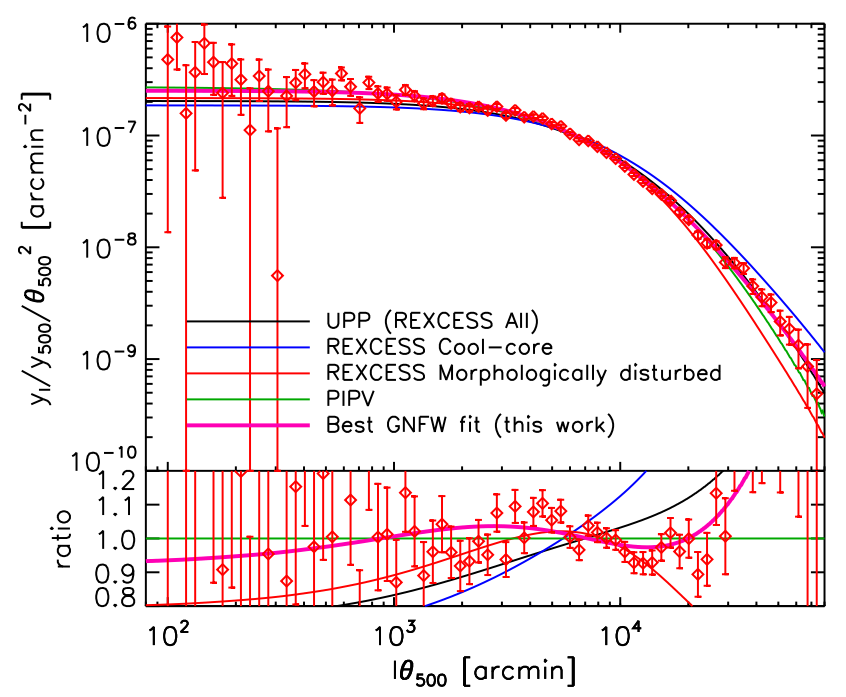

Figure 2. Best fit (thick solid pink line) of the average pressure profile of the SPT-SZ clusters in harmonic space compared to published pressure profiles. The red diamonds are the measurements from the joint analysis (identical to Figure 1). The solid green line is the PIPV profile and the black solid line the UPP. The cool-core (solid blue line) and morphologically disturbed (solid red line) profiles based on subsamples of the UPP are also displayed.

(multipole $l$ ) space. Our measurements are thus presented as $y_{l} / y_{500} / \theta_{500}^{2}$ vs. $l \theta_{500}$ (harmonic space) instead of the common $y(\theta) / y_{500}$ vs. $\theta / \theta_{500}$ (real space), where $\theta$ is the angular radius. The correspondance between $l \theta_{500}$ and $\theta / \theta_{500}$ can be established using the relation $l \sim \frac{180}{\theta[\mathrm{deg}]}$, so $l \theta_{500}[\operatorname{arcmin}] \sim 10800 \frac{\theta_{500}}{\theta}$.

We first cut $10 \times 10$ deg tangential maps centered on each of the 461 candidates for the six +three Planck and SPT-SZ maps. We applied an internal linear combination (ILC) [8] to produce a Compton $y$-map for each candidate. The ILC works in Fourier space and allows one to take into account the point spread function (PSF) of each map and the FTF of SPT. We corrected each map for the pixel window function and divided by $\theta_{500}^{2} y_{500}$. We then bin averaged in $\|\vec{k}\| \theta_{500}$ bins using inverse variance weights. This bin averaging step performs simultaneously the azimutal averaging and the spatial rescaling of the profiles. We note that before applying the ILC to the maps, we rescaled each SPT-SZ map by a factor depending on the mass and redshift of the cluster to take into account the Malmquist bias of the clusters in the SPT-SZ maps. The detailed description of the computation of this rescaling factor, together with a full explanation of the method, is given in Melin et al. [9].

\section{Results}

\subsection{Joint Planck and SPT-SZ pressure profile}

Results for the full sample are presented in Figure 1. The average profile extracted from Planck only, SPT-SZ only and Planck+SPT-SZ jointly are in very good agreement. They are also in very good agreement with the profile determined from Planck and XMM-Newton data on 62 nearby massive clusters [PIPV, 10]. Planck data provides information at large angular 


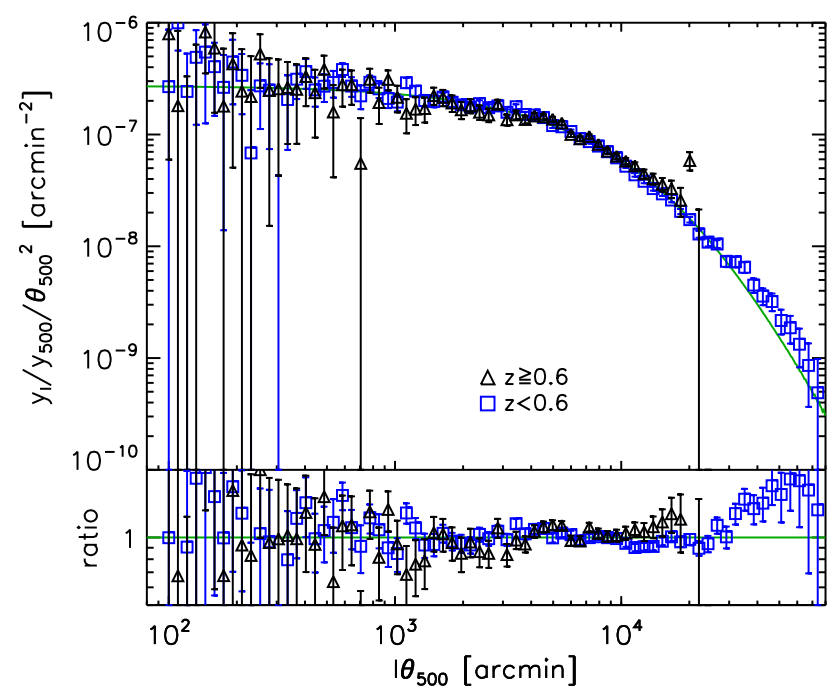

Figure 3. Evolution of the pressure profile of the SPT-SZ clusters. Blue squares (resp. black triangles) show measurements for the low (resp. high) redshift $(z<0.6)$ sample. The solid green line is the PIPV profile as in Figure 1 and 2.

scales (low multipole $l$ ) while the SPT-SZ data provides information at small angular scales (high multipoles). In particular, Planck outperforms SPT-SZ for scales $l \theta_{500}<2500$ arcmin and SPT-SZ outperforms Planck for $l \theta_{500}>6500$ arcmin.

In Figure 2, we present the best fit of the joint pressure profile (solid pink line). It is close to the PIPV profile (solid green line) at low $l \theta_{500}$ (outer part of the profile in real space) and close to the Universal Pressure Profile [UPP, 6] (black) at high $l \theta_{500}$ (the inner part of the profile in real space). The differences between our best-fitting profile, the UPP, and the PIPV at high $l \theta_{500}$ (inner part of the profile in real space) are smaller than the difference between the cool-core (solid blue line) and morphologically disturbed (solid red line) profiles.

\subsection{Evolution with redshift}

We split our sample in two at $z=0.6$ (close to the median redshift $z=0.56$ ) and studied the pressure profile of the two subsamples. Results are shown in Figure 3. The profile of the low redshift sample (blue squares) is in good agreement with the profile of the high redshift sample (black triangles). They are both in agreement with the PIPV profile. We also note that the high redshift sample lacks data points at $l \theta_{500}>2 \times 10^{4}$ arcmin due to the limited resolution of the SPT-SZ survey for high redshift $(z \geqslant 0.6)$ cluster profile.

\subsection{The cluster outskirts}

We now study the cluster outskirts. We projected the data points onto a grid and used the inverse fast Fourier transform to obtain a two dimensional Compton $y$-parameter profile. We obtained the envelope error by Monte Carlo sampling. The two dimensional profile is in good agreement with the PIPV and the best fit profiles. Aung et al. [11], Baxter at al. [12] and Zhang et al. [13] predicted that an accretion shock should be visible in the stacked profiles 
of relaxed clusters at a characteristic scale of $\theta / \theta_{200 \mathrm{~m}} \sim 2$ (equivalent to $\theta / \theta_{500} \sim 3$ ). We do not detect any obvious characteristic scale in this first study but we do not have dynamical state indicators for the cluster sample so we cannot select relaxed clusters. Moreover, we performed the average in $\theta_{500}$ and not in $\theta_{200 \mathrm{~m}}$. It is also possible that the sensitivities of the experiments are not sufficient to measure the effect. We will perform additional tests for the cluster outskirts in Melin et al. [9].

\section{Conclusion}

We presented the average pressure profile based on a joint analysis of Planck and SPT-SZ data for a sample of 461 clusters, a number much larger than previous analyses which includes tens of clusters. For the first time, we proposed to work and constrain the pressure profile in harmonic space, which is better suited for averaging profiles spanning a large range of mass and redshift. The measured profile is close to the pressure profile determined by the Planck collaboration on a sample of 62 clusters [10] in the outer regions. It confirms the excess of pressure at large radius $\left(\theta>\theta_{500}\right)$ with respect to the Universal Pressure Profile [6]. In the inner regions, our measured profile is in very good agreement with the Universal Pressure Profile. Dividing our sample into low and high redshift bins, we do not find a strong evolution. However, we lack some information in the inner part of the profile for the high redshift $(z \geqslant 0.6)$ subsample due to the limited resolution of the experiments. This fact reinforces the importance of high resolution ground based SZ experiments such as NIKA2, which complements the missing information of space-based and ground-based SZ surveys. We also studied the outskirts of the average profile. The average two dimensional Compton $y$-profile features multiple pressure drops at scales $\theta / \theta_{500}>2$ but we do not detect an obvious characteristic scale due to the accretion shock in this first study. We will perform additional tests in a forthcoming publication [9].

\section{Acknowledgements}

We would like to thank the anonymous referee for his/her useful comments and suggestions which helped to improve this proceeding.

\section{References}

[1] R.A. Sunyaev, Y.B. Zeldovich, Comments on Astrophysics and Space Physics 2, 66 (1970)

[2] R.A. Sunyaev, Y.B. Zeldovich, Comments on Astrophysics and Space Physics 4, 173 (1972)

[3] R. Chown, Y. Omori, K. Aylor, B.A. Benson, L.E. Bleem, J.E. Carlstrom, C.L. Chang, H.M. Cho, T.M. Crawford, A.T. Crites et al., ApJS239, 10 (2018), 1803. 10682

[4] L.E. Bleem, B. Stalder, T. de Haan, K.A. Aird, S.W. Allen, D.E. Applegate, M.L.N. Ashby, M. Bautz, M. Bayliss, B.A. Benson et al., ApJS216, 27 (2015), 1409.0850

[5] J.B. Melin, J.G. Bartlett, P. Tarrío, G.W. Pratt, A\&A647, A106 (2021), 2009.08822

[6] M. Arnaud, G.W. Pratt, R. Piffaretti, H. Böhringer, J.H. Croston, E. Pointecouteau, A\&A517, A92 (2010), 0910. 1234

[7] K.M. Górski, E. Hivon, A.J. Banday, B.D. Wand elt, F.K. Hansen, M. Reinecke, M. Bartelmann, ApJ622, 759 (2005), astro-ph/0409513

[8] M. Remazeilles, J. Delabrouille, J.F. Cardoso, MNRAS410, 2481 (2011), 1006.5599 
[9] J.B. Melin, G.W. Pratt, M. Arnaud, in prep. (2022)

[10] Planck Collaboration, P.A.R. Ade, N. Aghanim, M. Arnaud, M. Ashdown, F. AtrioBarandela, J. Aumont, C. Baccigalupi, A. Balbi, A.J. Banday et al., A\&A550, A131 (2013), 1207.4061

[11] H. Aung, D. Nagai, E.T. Lau, arXiv e-prints arXiv:2012.00977 (2020), 2012 . 00977

[12] E.J. Baxter, S. Adhikari, J. Vega-Ferrero, W. Cui, C. Chang, B. Jain, A. Knebe, arXiv e-prints arXiv:2101.04179 (2021), 2101.04179

[13] C. Zhang, I. Zhuravleva, A. Kravtsov, E. Churazov, arXiv e-prints arXiv:2103.03850 (2021), 2103.03850 